\title{
Transport, electronic, and structural properties of nanocrystalline $\mathrm{CuAlO}_{2}$ delafossites
}

\author{
O. J. Durá, ${ }^{1,2}$ R. Boada, ${ }^{3,4}$ A. Rivera-Calzada, ${ }^{5}$ C. León, ${ }^{5}$ E. Bauer, ${ }^{2}$ M. A. López de la Torre, ${ }^{1}$ and J. Chaboy ${ }^{3,4}$ \\ ${ }^{1}$ Departamento de Física Aplicada and INEI, Universidad de Castilla-La Mancha, ES-13071 Ciudad Real, Spain \\ ${ }^{2}$ Institute of Solid State Physics, Vienna University of Technology, AT-1040 Wien, Austria \\ ${ }^{3}$ Instituto de Ciencia de Materiales de Aragón, Consejo Superior de Investigaciones Científicas, \\ CSIC-Universidad de Zaragoza, ES-50009 Zaragoza, Spain \\ ${ }^{4}$ Departamento de Física de la Materia Condensada, Universidad de Zaragoza, ES-50009 Zaragoza, Spain \\ ${ }^{5}$ GFMC, Departamento de Física Aplicada III, Facultad de Física, Universidad Complutense de Madrid, ES-28040 Madrid, Spain
}

(Received 2 June 2010; revised manuscript received 21 September 2010; published 26 January 2011)

\begin{abstract}
This work reports on the effect of grain size on the electrical, thermal, and structural properties of $\mathrm{CuAlO}_{2}$ samples obtained by solid-state reaction combined with ball milling. Electrical characterization made in microcrystalline and nanocrystalline samples shows that the electrical conductivity decreases several orders of magnitude for the nanocrystalline samples, and, in addition, there is a large discrepancy between the activation energies associated to thermoelectric power $E_{S}$. The study of the $\mathrm{Cu} K$-edge x-ray absorption spectra of the $\mathrm{CuAlO}_{2}$ samples shows that the local structure around $\mathrm{Cu}$ is preserved after the sintering process, indicating that the observed behavior of the electrical conductivity is of intrinsic origin. Complex conductivity measurements as a function of frequency allow us to discard grain-boundaries effects on the electrical transport. Thus, the changes in $\sigma(T)$ and $S(T)$ are interpreted in terms of charge localization in the framework of small polarons. This is in agreement with the analysis of the near-edge region of the absorption spectra, which indicates that sintering favors the $\mathrm{Cu}-\mathrm{O}$ hybridization. As a consequence, oxygen atoms progressively lose their capability of trapping holes, and the electrical conductivity is also enhanced.
\end{abstract}

DOI: 10.1103/PhysRevB.83.045202

PACS number(s): 72.80.Jc, 71.38.Ht, 61.05.cj, 78.70.Dm

\section{INTRODUCTION}

Interest in thermoelectric materials is currently associated with their possible applications as power generators and refrigerators. They may be used to directly convert waste heat in electricity as produced in automobile engines, nuclear and fossil fuel energy plants, etc. Several materials have been proposed as potential candidates for thermoelectric applications, including skutterudites, ${ }^{1}$ clathrates, ${ }^{2}$ and half-Heusler alloys. ${ }^{3}$ Recently, new oxide materials have attracted great attention as promising thermoelectric materials for high-temperature power generation because of their high thermal stability, low toxicity, and oxidation resistance. ${ }^{4}$

Efficient thermoelectric materials must display high values of thermopower $(S)$ and electrical conductivity $(\sigma)$, combined with a low thermal conductivity $(\kappa)$. Therefore, the simplest strategy to improve efficiency in a material showing high thermopower values would be to decrease its thermal conductivity without changing, if possible, either $S$ or $\sigma$ values. Despite the fact that it has been reported to be successful in some cases ${ }^{5}$ this is not an easy task as a substantial reduction in $\kappa$ is usually accompanied by the reduction of $\sigma$. The reduction of $\kappa$ can be accomplished easily by introducing defects, reducing the grain size, or engineering low-dimensional materials. ${ }^{6}$ This opens the possibility of modifying the thermoelectric properties of traditional materials by applying, for example, a mechanical milling treatment.

The delafossite oxide $\mathrm{CuAlO}_{2}$ is a $p$-type transparent semiconductor $^{7}$ that could be considered as a candidate material for thermoelectric applications. It shows very high $S(\sim 400 \mu \mathrm{V} / \mathrm{K})$, moderate $\sigma\left(10^{-2} \mathrm{~s} \mathrm{~cm}^{-1}\right)$, and rather high $\kappa(\sim 10 \mathrm{~W} / \mathrm{mK})$ values at room temperature. ${ }^{8}$ Transparent conductors are being used in the window layers of solar cells, as front electrodes in flat-panel displays (FPD), defrosting windows in refrigerators or airplanes, and other technological applications. ${ }^{9}$ As a result of the peculiarities of the crystallographic structure, its electrical transport, mainly associated to the hopping of $\mathrm{Cu}$-site holes, ${ }^{10}$ is highly anisotropic and, moreover, it is generally accepted that the electrical conductivity in this system is due to small polarons. ${ }^{11-13}$ However, $\mathrm{CuAlO}_{2}$ has been reported to exhibit not only small polaron transport, ${ }^{8}$ but also variable-range hopping ${ }^{14}$ and band conduction. ${ }^{15-17}$ Since most reported experiments were performed on thin film and bulk polycrystalline materials, these results are likely to be influenced by grain boundaries, strain, and other defects common in such materials. Consequently, the origin of the $p$-type conductivity of the undoped $\mathrm{CuAlO}_{2}$ is still controversial.

In this paper, we present a study of the effect of decreasing the grain size, down to $18 \mathrm{~nm}$, on the electrical, thermal, and structural properties of $\mathrm{CuAlO}_{2}$. It is expected that, by decreasing the grain size, the thermal conductivity also decreases, thus improving the thermoelectric efficiency. Therefore, we have performed a comparative study of $S(T)$ and $\sigma(T)$ on microcrystalline and nanocrystalline samples of $\mathrm{CuAlO}_{2}$ in which the reduction of the grain size is obtained through ball milling. The role of the grain boundaries in electrical-transport properties has been determined from the measurement of the complex conductivity as a function of frequency. In addition, specific heat and thermal conductivity measurements have been carried out. $\mathrm{Cu} K$-edge x-ray absorption spectroscopy experiments have been performed to determine if either the $\mathrm{Cu}$ electronic state or the local structure of the $\mathrm{Cu}$ ions is modified by the milling process. The observed changes in $S(T)$ and $\sigma(T)$ induced by the reduced grain size have been discussed in terms of the polaron theory for electronic transport according to the current models for electrical conduction in this type of material. 


\section{EXPERIMENTAL AND COMPUTATIONAL METHODS}

The microcrystalline and nanocrystalline samples of $\mathrm{CuAlO}_{2}$ studied in this work were prepared by means of a conventional solid-state ceramic method ${ }^{7}$ consisting of repeated grinding and calcining steps of the stoichiometric mixture of the $\mathrm{Cu}_{2} \mathrm{O}$ and $\mathrm{Al}_{2} \mathrm{O}_{3}$ starting powders. In order to reduce the grain size, the powders obtained after the solid-state reaction were subjected to mechanical milling for 36 hours. The milling process was carried out in a Retsch PM400 planetary ball mill equipped with zirconia pots and balls, at a speed of $250 \mathrm{rpm}$, in air. The ball and powder weight ratio was $7: 1$. To monitor the alloying process, $\mathrm{x}$-ray diffraction patterns were recorded for different milling times, using an Xpert (Philips) diffractometer. Powders obtained from the milling process (denoted as $B M$ ) were uniaxially cold pressed into pellets $(10 \mathrm{~mm}$ diameter and $1.5 \mathrm{~mm}$ thick) by applying a pressure of $1 \mathrm{GPa}$. Then, two samples were prepared by sintering the milling powders at two different temperatures: (i) $T_{S}=550{ }^{\circ} \mathrm{C}$, which is insufficient to promote the grain growth, obtaining a sample with a grain size of $18 \mathrm{~nm}$ (hereafter denoted as NANO), and (ii) $T_{S}=1100^{\circ} \mathrm{C}$, for which the sample (hereafter denoted as $M I C R O)$ displays a grain size of $0.34 \mu \mathrm{m}$ as estimated from atomic-force-microscope (AFM) images (not shown). The Scherrer formula, corrected by instrumental broadening, was used to estimate the value of the grain size in the NANO and $B M$ samples, displaying no difference between the two. The density of the compacts was determined following the Archimedes method using water as an immersion fluid. The density is $4.96 \mathrm{~g} \mathrm{~cm}^{-3}$ for the MICRO sample and $4.43 \mathrm{~g} \mathrm{~cm}^{-3}$ for the NANO sample, which means $97 \%$ and $87 \%$ of the theoretical density, respectively.

Thermopower and dc electrical measurements were performed by using a commercial MMR-technologies system in the temperature range between 130 and $650 \mathrm{~K}$. The electrical measurements were performed on $5 \times 5$-mm flat plates by using the Van der Pauw method. The thermopower $S(T)$ was obtained in $1 \times 1 \times 8-\mathrm{mm}$ samples by comparison with a reference constantan wire. The reproducibility of these experiments was verified by repeating the measurements using new contacts both on the sample and on the reference constantan wire. The estimated experimental accuracy of the $S(T)$ measurements shown in this work is better than $\pm 5 \%$.

Complex conductivity measurements were performed on a broadband dielectric spectrometer (BDS-80, Novocontrol) in the $10^{-2}-10^{7} \mathrm{~Hz}$ frequency range, with an applied ac voltage of $1 \mathrm{~V}$. Electrodes were deposited on the pellet's flat surfaces by applying platinum paste. The thermal conductivity $\kappa$ of each pellet was determined from separate measurements of the thermal diffusivity $\alpha$, specific heat $C_{p}$, and density $\rho$, using the relationship $\kappa=\rho \alpha C_{p}$. Thermal diffusivity was measured with a commercial Anter Flashline 3000 system. All the samples were covered with a graphite screen to ensure full absorption of the flash light at the front surface and highest emissivity from the back side. Thermal diffusivity was measured from 173 to $673 \mathrm{~K}$ in inert atmosphere. Several measurements were performed under the same conditions to verify that there were no changes in samples or damage of the graphite screen during the experiment. Thermal-diffusivity values were obtained after applying the Clarke and Taylor correction, ${ }^{18}$ which takes into account radiative losses occurring in nonadiabatic conditions. Specific heat was measured between 150 and $650 \mathrm{~K}$ with a Netzsch-Jupiter 404 differential scanning calorimeter (DSC) using platinum crucibles in helium atmosphere. A single-crystal sapphire sample was used as a reference. The thermal-conductivity values were corrected to account for the porosity $P=1-\rho / \rho_{\text {theor }}$ using the relation proposed by Klemens: $\frac{\kappa_{\text {porous }}}{\kappa_{\text {dense }}}=1-\frac{4}{3} P .{ }^{19}$

$\mathrm{Cu} K$-edge x-ray absorption measurements were performed at the BM25A SpLine beamline of the European Synchrotron Radiation Facility (ESRF). The storage ring was operated in the 16-bunch mode with a typical current of $90 \mathrm{~mA}$ at an electron beam energy of $6 \mathrm{GeV}$. Measurements were performed at room temperature in the transmission detection mode on homogeneous thin layers of the powdered samples. The beamline is equipped with a pseudo-channel-cut-type monochromator with two fixed $\mathrm{Si}(111)$ crystals in the $(-n,+n)$ configuration. Harmonic rejection was achieved by detuning the second crystal from the parallel alignment. The absorption spectra were analyzed according to standard procedures. ${ }^{20-22}$

The $a b$ initio computation of the $\mathrm{Cu} K$-edge XANES spectra was carried out by using the multiple-scattering code CONTINUUM ${ }^{23}$ based on the one-electron full-multiplescattering theory. ${ }^{24}$ A complete discussion of the procedure can be found elsewhere. ${ }^{25,26}$ During the computations, special attention has been given to the choice for the exchange and correlation part of the final-state potential ${ }^{27-29}$ and to the need of using more than a single absorption channel in accounting for the $\mathrm{Cu} K$-edge absorption. ${ }^{30-33}$ In all the cases, the theoretical spectra have been convoluted with a Lorentzian shape function to account for the core-hole lifetime $(\Gamma=1.5 \mathrm{eV})$ (Ref. 34) and the experimental resolution $(\Gamma=1 \mathrm{eV})$.

\section{RESULTS AND DISCUSSION}

The x-ray diffraction (XRD) patterns of the samples $N A N O$ and $M I C R O$ sintered at $T_{S}=550^{\circ} \mathrm{C}$ and $1100^{\circ} \mathrm{C}$, respectively, are shown in Fig. 1(a). Both samples present the corresponding delafossite structure (rhombohedral space group $\mathrm{R} \overline{3} \mathrm{~m}$ ). The lattice parameters calculated from the X-ray diffraction patterns are $a=2.859 \AA$ and $c=16.942 \AA$ for the MICRO sample and $a=2.861 \AA$ and $c=16.955 \AA$ for the NANO sample. The XRD patterns show sharp peaks for the sample sintered at high temperature, indicative of a microcrystalline sample, while those of the sample sintered at low temperature display the broad peaks characteristic of nanocrystalline materials. Indeed, the dependence of the XRD patterns as a function of the milling time [see inset of Fig. 1(a)] shows a progressive peak broadening, in agreement with the expected grain-size reduction during the milling process. The quantitative Rietveld analysis corresponding to the $M I C R O$ sample, reported in Fig. 1(b), indicates the presence of a small amount, $\lesssim 5 \%$, of $\mathrm{CuAl}_{2} \mathrm{O}_{4}$ (cubic space group $\mathrm{Fd} \overline{3} \mathrm{~m}$ ).

\section{A. Transport properties}

The results of dc electrical conductivity measurements performed on the sintered $\mathrm{CuAlO}_{2}$ samples are shown as $T \sigma$ 

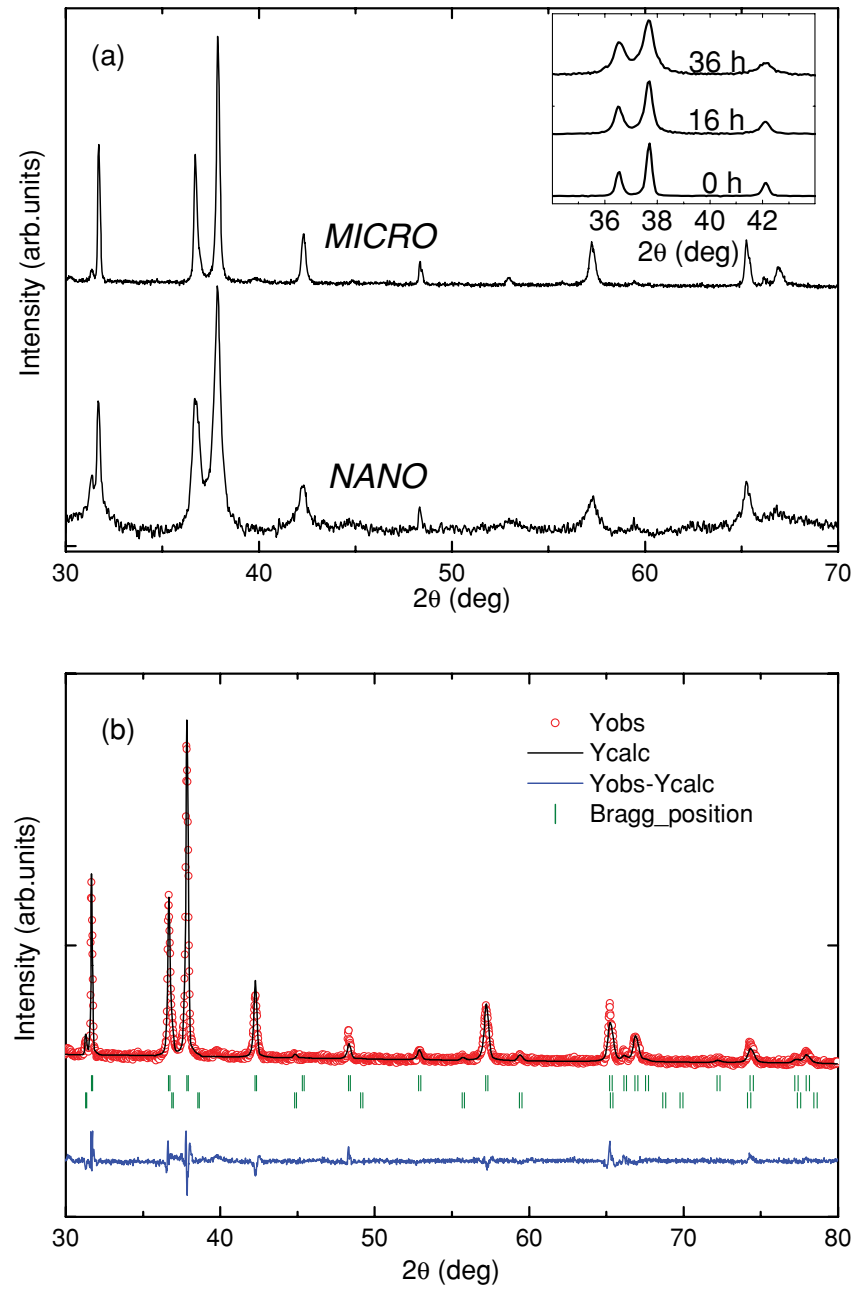

FIG. 1. (Color online) (a) Comparison of the x-ray diffraction patterns for the microcrystalline and nanocrystalline samples. The evolution of the milling process for 0,16 , and $36 \mathrm{~h}$ is shown in the inset. (b) x-ray diffraction pattern for the MICRO sample. The solid line corresponds to the profile calculated from the Rietveld refinement, and $Y_{\mathrm{obs}}-Y_{\text {calc }}$ is the intensity difference between experimental data and Rietveld calculations.

versus $1000 / T$ in Fig. 2. The nanocrystalline sample exhibits a much lower electrical conductivity (more than three orders of magnitude smaller) than the microcrystalline sample. Indeed, the observed temperature dependence is in agreement with the expected one if electrical conduction is dominated by polaronic carriers. ${ }^{35}$ At high temperature, typically above $\theta_{\mathrm{D}} / 2$, where $\theta_{\mathrm{D}}$ denotes Debye temperature, the electrical conductivity is thermally activated, i.e.,

$$
T \sigma(T)=\sigma_{0} e^{-E_{\sigma} / k_{\mathrm{B}} T} .
$$

By contrast, a crossover to variable-range hopping (VRH) occurs below $\theta_{\mathrm{D}} / 2$, for which Mott's law ${ }^{35}$ predicts

$$
\sigma(T) \propto e^{\left(T_{0} / T\right)^{1 / 4}} .
$$

From our own specific heat measurements (see the following), we estimate $\theta_{\mathrm{D}} / 2 \sim 300 \mathrm{~K}$ for both samples. These values are in good agreement with the results displayed in Fig. 2, showing a smooth crossover from the high-temperatureactivated behavior $(350-650 \mathrm{~K})$ to the low-temperature VRH

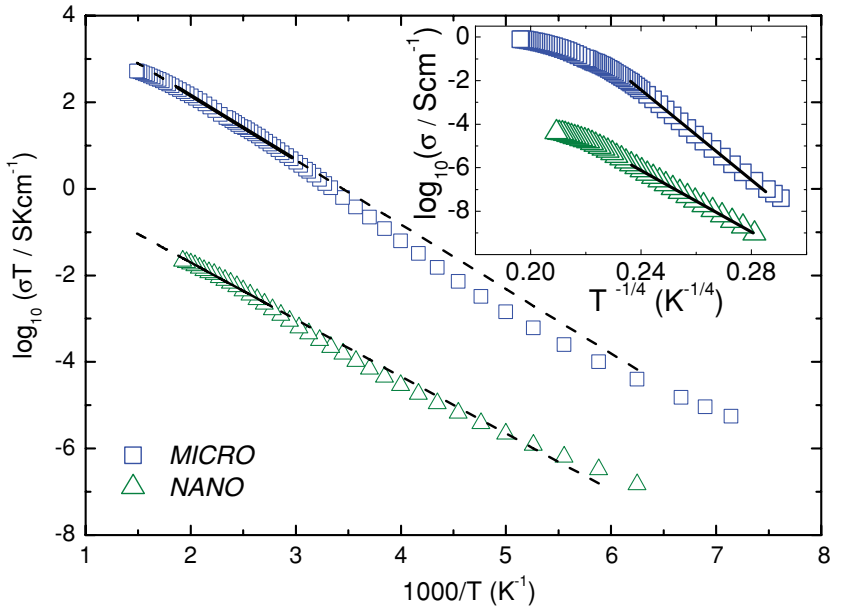

FIG. 2. (Color online) $T \sigma(T)$ as a function of inverse temperature for the microcrystalline (blue, open square) and nanocrystalline samples (green, open triangle). Solid lines represent fits to Arrhenius law. The inset shows the same data plotted as $\sigma(T)$ vs $T^{-1 / 4}$. Solid lines are fits to Eq. (2).

regime (140-300 K). From the fits of the high-temperature data (above $T=350$ and $370 \mathrm{~K}$ for the $M I C R O$ and $N A N O$ samples, respectively) to Eq. (1), we obtain values of the conductivity activation energies $E_{\sigma}=295 \mathrm{meV}$ and $E_{\sigma}=$ $260 \mathrm{meV}$ for the $M I C R O$ and NANO samples, respectively. Fits of the low-temperature data to the VRH law are shown in the inset of Fig. 2.

Complementary complex impedance measurements were made in the same temperature range, with the aim to elucidate whether, in both samples, the dc measurements correspond to the bulk electrical conductivity and, consequently, whether the reduced values obtained for the nanocrystalline sample are caused by carrier blocking at grain boundaries. Complex conductivity as a function of frequency, i.e., $\sigma^{*}(\omega)=\sigma^{\prime}(\omega)+$ $j \omega \epsilon_{0} \epsilon^{\prime}(\omega)$, was directly obtained from our measurements, with $\sigma^{\prime}(\omega)$ its real part, $\epsilon_{0}$ the permittivity of the vacuum, and $\epsilon^{\prime}(\omega)$ the real part of the complex dielectric permittivity. Figure 3 shows the frequency dependence of the real part of conductivity for the MICRO [Fig. 3(a)] and NANO [Fig. 3(b)] samples at several fixed temperatures. In the case of the $N A N O$ sample, the data clearly show the universal ac response. Consequently, the frequency dependence of the conductivity can be well described using the Jonscher's expression ${ }^{36}$

$$
\sigma^{*}(\omega)=\sigma_{\mathrm{dc}}\left[1+\left(j \omega / \omega_{p}\right)^{n}\right],
$$

where $\omega_{p}$ is the characteristic frequency and $n$ takes values $\leqslant 1$ and quantifies the dispersive behavior. This power-law dependence of the ac conductivity appears at low temperatures and relatively high frequencies. Similar behavior is found in ion-conducting solids, amorphous and polycrystalline semiconductors, and polymers or transition metal oxides, among others, ${ }^{36-38}$ while it is not applicable for metallic or delocalized systems. In the case of the MICRO sample, the dispersion region is shifted toward higher frequencies and lower temperatures as a consequence of the higher $\sigma_{\mathrm{dc}}$ values displayed. In the $N A N O$ sample, the charge carriers are more localized, and therefore the relaxation time is longer (lower frequencies) than in the MICRO sample. This explains the observed shift of the 

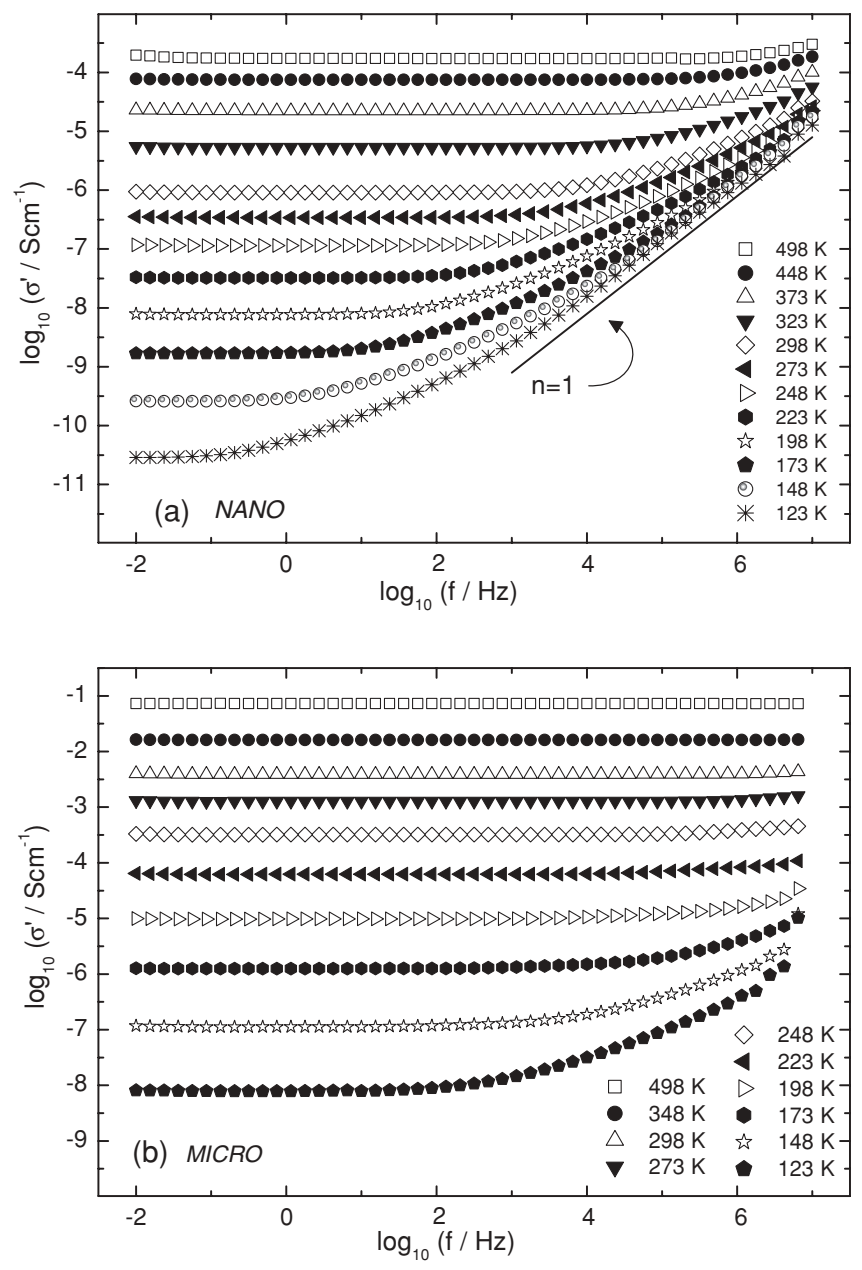

FIG. 3. Real part of conductivity $\left(\sigma^{\prime}\right)$ vs frequency of the (a) $N A N O$ and (b) MICRO samples.

dispersion region toward lower values of $\omega_{p}$. It is important to note that, in both cases, the plateau that corresponds to $\sigma_{\mathrm{dc}}$ observed at frequencies lower than $\omega_{p}$ shows a constant value down to lower frequencies. Thus, there is no indication of the existence of blocking effects of charge carriers at grain boundaries. Moreover, the values of $\sigma_{\mathrm{dc}}$ obtained from the ac conductivity measurements are in good agreement with those obtained using the Van der Pauw method.

The results of thermopower measurements on the MICRO and NANO samples are shown in Fig. 4. The high-temperature $S(T)$ values are similar to those reported for polycrystalline $\mathrm{CuAlO}_{2}$ by different authors. ${ }^{8,39}$ However, the $S(T)$ of the nanocrystalline sample displays a less marked temperature dependence than that of the microcrystalline sample. If the charge carriers were polarons, the thermopower is predicted to follow the law ${ }^{35}$

$$
S(T)=\frac{k_{\mathrm{B}}}{e}\left(\frac{E_{S}}{k_{\mathrm{B}} T}+\alpha\right),
$$

where $E_{S}$ is the thermopower activation energy and $\alpha$ is a constant associated with the spin and mixing entropy. ${ }^{40}$ It is clear from Fig. 4 that $E_{S}$ is much smaller for the nanocrystalline sample than for the microcrystalline sample. Indeed, the fits of our data to Eq. (4), performed for each sample in the same tem-

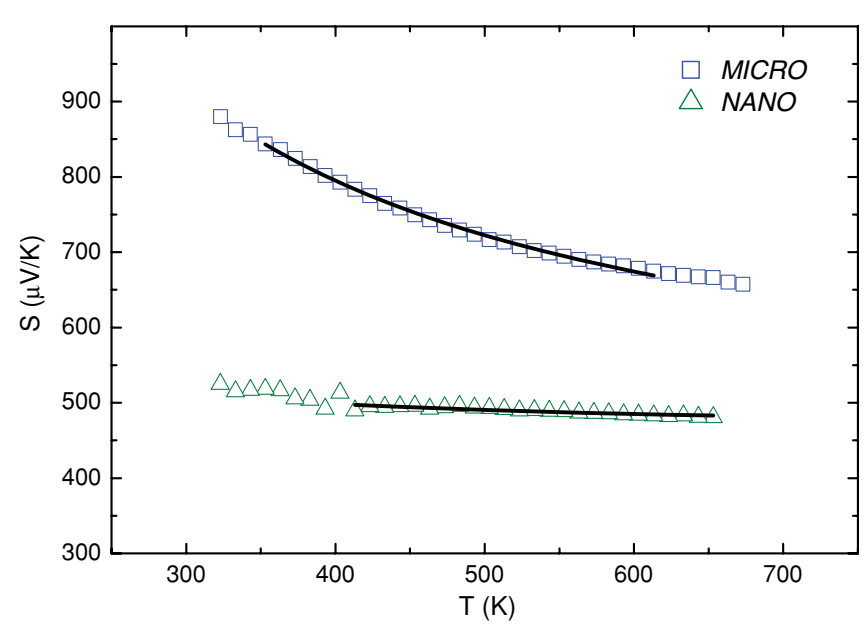

FIG. 4. (Color online) Temperature dependence of the Seebeck coefficient for the MICRO (blue, open square) and NANO (green, open triangle) $\mathrm{CuAlO}_{2}$ samples. Solid lines are fits to Eq. (4) in the same temperature range as those of electrical conductivity (see text for details).

perature range as those of the high-temperature electrical conductivity, yield $E_{S}=153 \mathrm{meV}$ and $\left(k_{\mathrm{B}} / e\right) \alpha=412.3 \mu \mathrm{V} / \mathrm{K}$ for the MICRO sample and $16 \mathrm{meV}$ and $460.2 \mu \mathrm{V} / \mathrm{K}$, respectively, for the $N A N O$ sample. These results suggest a change of the charge carriers' localization. Within a small polaron framework, ${ }^{11,12,40}$ the measured activation energy $E_{\sigma}$ is the sum of the energy required to jump in a given direction $W_{H}$, and the activation needed to create the carriers $\epsilon_{0}$, whereas $E_{S}$ is the energy required to generate the carriers. Therefore, $E_{\sigma} \equiv \epsilon_{0}+W_{H}-J$, where $J$ is the transfer integral and $\epsilon_{0} \equiv E_{S}$. The activation energies of $E_{\sigma}$ and $E_{S}$ are obtained from the fitting of the conductivity and thermopower curves to the expressions given in Eqs. (1) and (4). Thus, the value of $W_{H}$ is obtained as $E_{\sigma}-E_{S}$ assuming that, as usual, $J \ll W_{H} \cdot{ }^{40}$ Table I summarizes the activation energies and the polaron hopping energies. The large discrepancy between the activation energies for conductivity $E_{\sigma}$ and thermoelectric power $E_{S}$ is a typical feature of polaronic transport. ${ }^{41}$ In view of these results, the experimentally observed changes in $\sigma(T)$ and $S(T)$ as a function of the grain size can be attributed to the decreasing of the width of the polaron band gap, which leads to an increased localization of the charge carriers. ${ }^{42}$

In addition, the thermal properties of the samples have been studied by determining the thermal conductivity from independent specific heat, thermal diffusivity, and density measurements. We show in Fig. 5(a) the temperature dependence of the molar specific heat $C_{p}$ between 160 and $665 \mathrm{~K}$ for both $M I C R O$ and $N A N O$ samples. The specific heat at high temperature reaches the values predicted by the Dulong and Petit law $\left(99.76 \mathrm{~J} \mathrm{~mol}^{-1} \mathrm{~K}^{-1}\right)$. It should be noted that

TABLE I. Activation energies $E_{\sigma}, E_{S}$, and $W_{H}$ for microcrystalline and nanocrystalline $\mathrm{CuAlO}_{2}$ samples.

\begin{tabular}{lccc}
\hline \hline Sample & $E_{\sigma}(\mathrm{meV})$ & $E_{S}(\mathrm{meV})$ & $W_{H}(\mathrm{meV})$ \\
\hline NANO & $260 \pm 20$ & $16 \pm 1$ & $244 \pm 20$ \\
MICRO & $295 \pm 10$ & $153 \pm 5$ & $142 \pm 15$ \\
\hline \hline
\end{tabular}



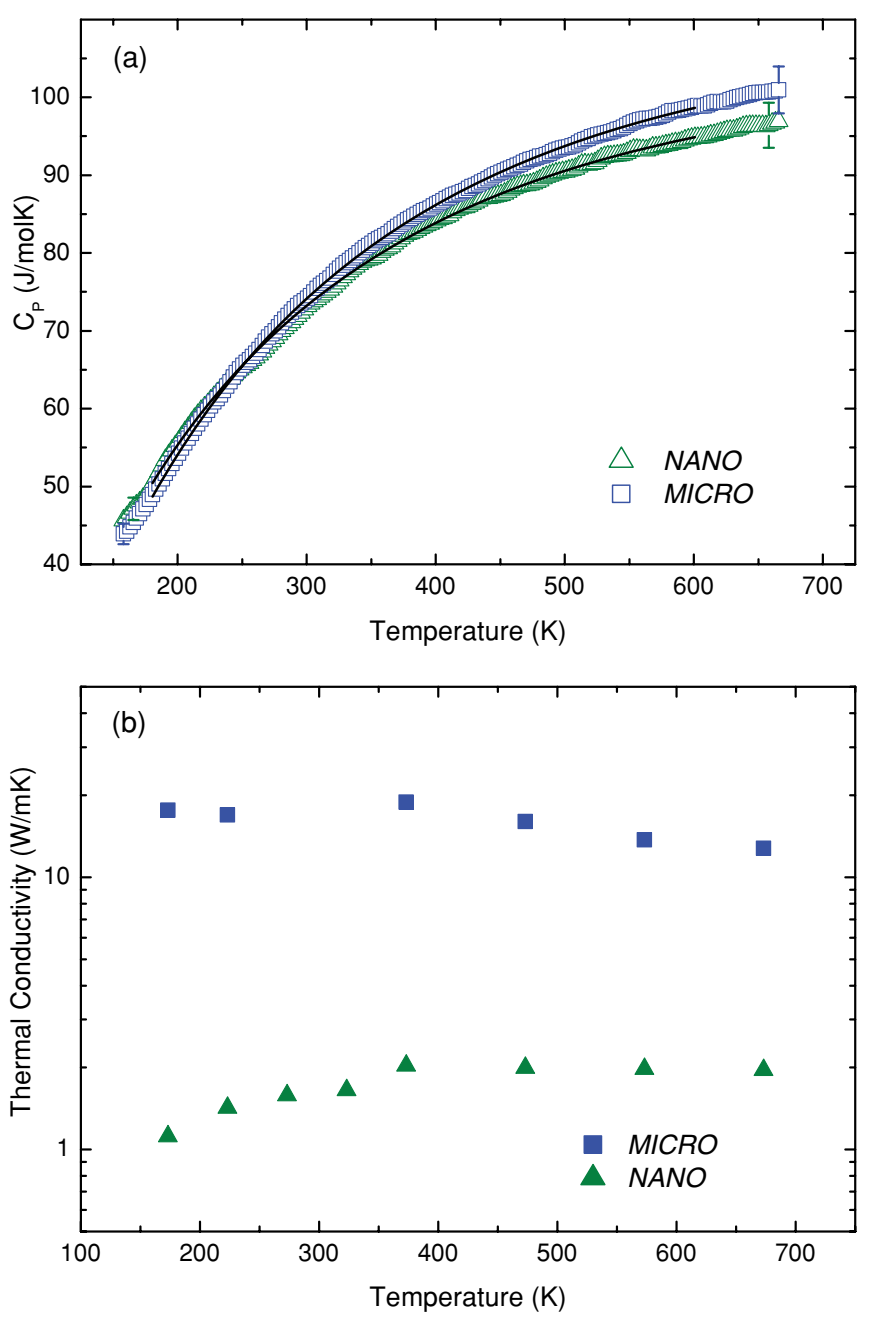

FIG. 5. (Color online) (a) Temperature dependence of the specific heat for microcrystalline (blue, open square) and nanocrystalline (green, open triangle) $\mathrm{CuAlO}_{2}$ samples. Solid lines correspond to the fits described in Ref. 43 (see text for details). (b) Temperature dependence of the thermal conductivity for microcrystalline (blue, filled square) and nanocrystalline (green, filled triangle) $\mathrm{CuAlO}_{2}$ samples.

the observed differences for both samples are smaller than the equipment accuracy, $\pm 3 \%$, which we have included as error bars in Fig. 5(a). Thus, we conclude that $C_{p}$ does not depend significantly on the grain size. In order to estimate the Debye temperature, we have performed an analysis similar to that described in Ref. 43, which assumes acoustic and optic contributions to the lattice specific heat. The results obtained by applying this model have been represented as solid lines in Fig. 5(a). The temperature dependence of the thermal conductivity $\kappa$ between 173 and $673 \mathrm{~K}$ for both MICRO and NANO samples is shown in Fig. 5(b). The raw data were corrected in order to take into account the porosity of the samples. For this purpose, different correction methods were applied. As different methods yielded very similar results, we have used the commonly used Klemens relation. ${ }^{19}$ As shown in Fig. 5(b), the thermal conductivity exhibits a strong dependence on the grain size. In addition, the nanocrystalline sample displays a significant reduction in the thermal conductivity with respect to that of the MICRO sample. As a matter of fact, the $\kappa$ values obtained for the case of the nanocrystalline sample above room temperature $\left(\sim 2 \mathrm{~W} \mathrm{~m}^{-1} \mathrm{~K}^{-1}\right)$ are of the same order than those reported for disordered crystals. ${ }^{44}$ A similar decrease of the thermal conductivity has been observed in other nanocrystalline materials ${ }^{45,46}$ and can be interpreted as a genuine effect of the grain boundaries over phonon transport. ${ }^{47}$

\section{B. Cu $K$-edge $x$-ray absorption spectroscopy}

The picture emerging from the transport characterization discards effects of the grain boundaries over the electrical transport. However, we can not a priori exclude that the observed behavior of the electrical conductivity is not associated to the presence of impurity phases or to chemical disorder in the grain boundaries. Therefore, we have performed a detailed X-ray absorption spectroscopy study at the $\mathrm{Cu} K$ edge in order to determine the effect of the sintering process, and thus of the grain size, in both the electronic state and the local structure of $\mathrm{Cu}$ atoms.

The comparison of the $\mathrm{Cu} K$-edge extended x-ray absorption fine structure (EXAFS) signals and their Fourier transforms (FT) for the three samples studied in this work is shown in Fig. 6. The EXAFS signals have been Fourier transformed by using a sine window in the $k$ range from 2.4 to $8.5 \AA^{-1}$. For the sake of accuracy, we have performed this comparison by using two different $k$ weights of the extracted EXAFS signals, i.e., $k \cdot \chi(k)$ and $k^{3} \cdot \chi(k)$. As shown in the figure, the EXAFS signals are similar for all the samples. They show the same spectral features, and the only appreciable difference is a slight reinforcement of the EXAFS amplitude as the sintering temperature increases. This behavior suggests that the local structure around the absorbing $\mathrm{Cu}$ is similar in all of the samples. This is made more evident in the comparison of the modulus of the Fourier transform shown in Figs. 6(c) and 6(d). The first peak in the FT is due to the two nearest-neighbor oxygen atoms $R_{\mathrm{Cu}-\mathrm{O}}=1.86 \AA$. The second peak between 2 and $4 \AA$ is due to the contributions of the second to fourth coordination shells $\left(6 \mathrm{Cu}, R_{\mathrm{Cu}-\mathrm{Cu}}=2.87 \AA\right.$; $6 \mathrm{Al}, R_{\mathrm{Cu}-\mathrm{Al}}=$ $3.28 \AA$; and $12 \mathrm{O}, R_{\mathrm{Cu}-\mathrm{O}}=3.42 \AA$ ) and to multiple-scattering (MS) effects. These two peaks were back-transformed into $k$ space by Fourier filtering in the $0.4 \leqslant R \leqslant 2 \AA$ and $2 \leqslant R$ $\leqslant 3.5 \AA$ ranges, respectively. Then, the difference of the local environment of $\mathrm{Cu}$ in the three studied samples was assessed by analyzing the filtered EXAFS signals. This was done by using the NPI fitting software. ${ }^{22}$ Amplitude and phase functions of the coordination spheres were calculated with the FEFF8 code (version 8.1). ${ }^{48}$ Moreover, the phase and amplitude transferability method ${ }^{20,49}$ also was used by considering the MICRO sample as the reference compound with the $\mathrm{CuAlO}_{2}$ delafossite structure. In both cases, the same $R_{\mathrm{Cu}-\mathrm{O}}$ interatomic distance was found for the annealed $N A N O$ and $M I C R O$ samples, whereas that of the $B M$ sample shows a $0.03-\AA$ contraction $\left(R_{\mathrm{Cu}-\mathrm{O}}=1.83 \pm 0.01 \AA\right)$. In addition, the Debye-Waller factor of both annealed samples increases with respect to that of the $B M$ sample by $\Delta \sigma^{2}=0.004 \pm 0.001 \AA^{2}$ and $0.006 \pm 0.001 \AA^{2}$ for the NANO and MICRO samples, respectively. By contrast, the behavior of the Debye-Waller factor is inverted regarding the EXAFS contribution of the second coordination sphere. It should be noted that (in this 

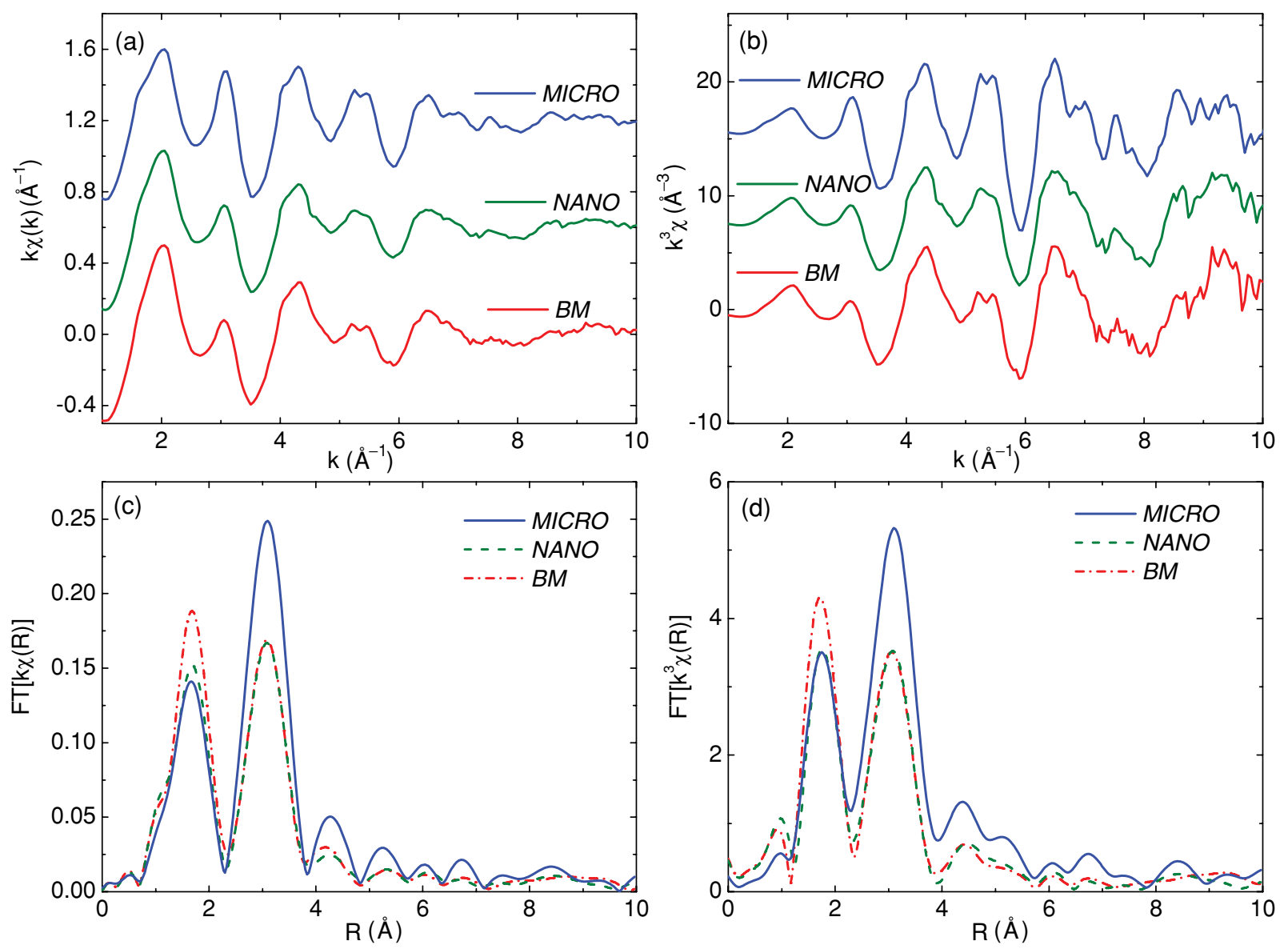

FIG. 6. (Color online) Comparison of the experimental EXAFS spectra (a) $k \cdot \chi(k)$ and (b) $k^{3} \cdot \chi(k) \mathrm{Cu} K$ edge for the samples studied in this work. Comparison of the Fourier transforms of the (c) $k \cdot \chi(k)$ and (d) $k^{3} \cdot \chi(k)$ spectra for the same samples [MICRO (blue, solid line), $N A N O$ (green, dash line), and $B M$ (red, dash-dot line)].

case, it is needed to add the EXAFS contribution of the second to fourth coordination shells) we have applied the phase and amplitude transferability method by considering an averaged single-shell interatomic distance of $3.21 \AA$ Å. In this way, we have found that the Debye-Waller factor increases by $\Delta \sigma^{2}=0.01 \pm 0.003 \AA^{2}$ for both $B M$ and $N A N O$ samples with respect to that of the $M I C R O$ reference sample. In several cases, the change in the local disorder could be the signature of polaron effects (see, for example, Refs. 50 and 51). However, the variation of the $\mathrm{Cu}-\mathrm{O}$ disorder as a signature of the presence of the polaron should be also reflected, contrary to our case, in the disorder of the further coordination shells $(\mathrm{Cu}-\mathrm{Cu}$, $\mathrm{Cu}-\mathrm{Al}$, etc.) showing the same trend in the modification of the width of the pair-distribution function $\left(\sigma^{2}\right)$ and of the interatomic distances..$^{51}$ On the contrary, our results indicate that the next-neighbor $\mathrm{Cu}$ environment is similar in all the samples. The sintering temperature affects the disorder of further coordination shells lying in the plane perpendicular to the $\mathrm{O}-\mathrm{Cu}-\mathrm{O}$ bond. In this respect, the EXAFS data indicate that the $\mathrm{O}-\mathrm{Cu}-\mathrm{O}$ is more rigid in the as-cast $B M$ sample and softens upon the sintering that, on the contrary, enhances the local order in the planes perpendicular to this bond. These results, and in particular the fact that disorder affects differently the observed FT peaks, are in agreement with the temperature effects in the EXAFS of delafossite structure compounds reported by Ahmed et al..$^{52}$ They show that the temperature effect is rather weak for the first-shell peak, indicating a relatively stiff link between nearest-neighbor copper and oxygen atoms, while it is weaker for the outer shells. It should be also noted that the FT modulus of the three samples exhibits the same peaks for $R \geqslant 4 \AA$. These peaks are associated to the interplay of further coordination shells and MS effects. All of these results point out that the observed changes in the electrical conductivity of the samples are not associated to the modification of the local structure around the $\mathrm{Cu}$ atoms.

In order to discern the origin, structural versus electronic, of the electrical conductivity behavior of these samples, we have studied both the near-edge and $\mathrm{x}$-ray absorption near-edge structures (XANES) regions of the $\mathrm{Cu} K$-edge absorption spectra. The near-edge region of the absorption spectrum is extremely sensitive to modifications of the density of states ${ }^{53,54}$ while the XANES part of the spectrum is related to the local structure around the absorbing atom. XANES is characterized by a higher sensitivity than EXAFS to the geometrical details of the absorbing site (overall symmetry, distances, and bond angles). Hence, this study can provide us a deeper insight into the occurrence of electronic (involving localization and hybridization phenomena) versus structural modifications ${ }^{55-58}$ associated to the sintering process in these 


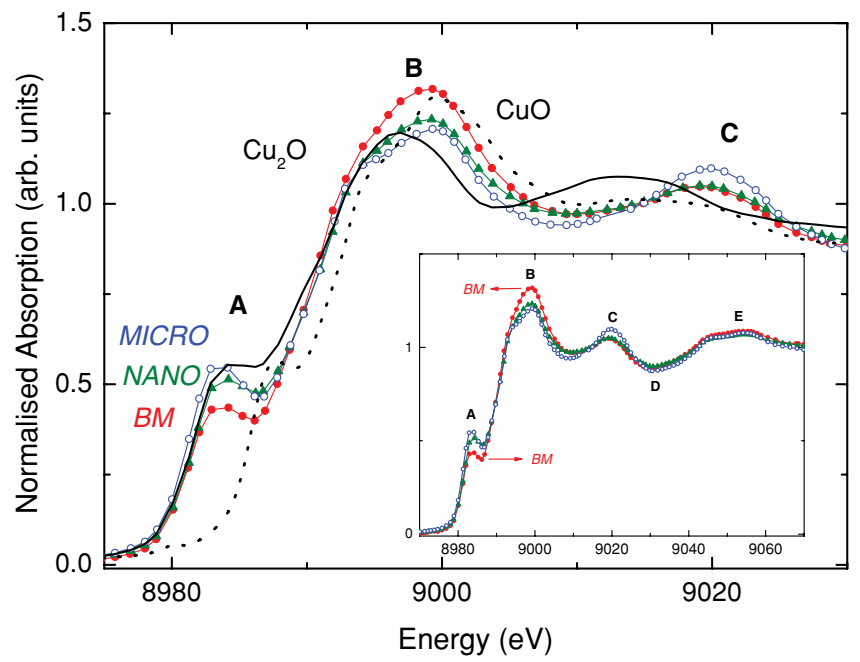

FIG. 7. (Color online) Comparison of the near-edge $\mathrm{Cu} K$-edge absorption of the $\mathrm{CuAlO}_{2}$ samples: $B M$ (filled bullet), NANO (filled triangle), and $M I C R O$ (open circle). The absorption of the reference compounds $\mathrm{Cu}_{2} \mathrm{O}$ (black, solid line) and $\mathrm{CuO}$ (black, dots) are also shown. In the inset, the comparison in an extended energy range of the $\mathrm{Cu} K$-edge absorption spectra of the $\mathrm{CuAlO}_{2}$ samples is shown.

systems. Moreover, as XANES is more sensitive than EXAFS to the bonding geometry, this study offers the possibility of detecting the increase of the impurity phases amount and/or of chemical disorder involving substitutional exchange of $\mathrm{Cu}$ and $\mathrm{Al}$ atoms during the mechanical milling.

The $\mathrm{Cu} K$-edge XANES spectra of the $\mathrm{CuAlO}_{2}$ samples and those of the reference compounds are displayed in Fig. 7. The references $\mathrm{Cu}_{2} \mathrm{O}$ and $\mathrm{CuO}$ correspond to compounds in which the formal valence of $\mathrm{Cu}$ is $\mathrm{Cu}(\mathrm{I})$ and $\mathrm{Cu}(\mathrm{II})$, respectively. This comparison indicates both that the electronic state of $\mathrm{Cu}$ in all the samples is close to $\mathrm{Cu}(\mathrm{I})$ and that this formal valence state does not change upon the sintering process. In addition, the shape and the energy position of the XANES spectral features ( $C$ to $E$ ) remain unchanged, and only the amplitude is modified. The fact that all the spectral features, maxima and minima, appear at the same energy position indicates that the local environment of $\mathrm{Cu}$ does not change upon sintering. The observed increase of the amplitude of the XANES oscillations for the sample sintered at the highest temperature (MICRO) suggests that the alloy becomes more ordered after the annealing treatment, in agreement with the EXAFS results.

It is worth noting that the $\mathrm{x}$-ray absorption spectra show significant differences at the near-edge region. The spectral shape of the $\mathrm{Cu} K$-edge spectra exhibits a shoulderlike feature at the absorption threshold $(A)$, reflecting the overlapping of the local $p$-projected density of states with the outer $s$ and $d$-symmetry orbitals. Because the $\mathrm{Cu} d$ states and $\mathrm{O}$ $p$ states hybridized, the modification of the width and the intensity of the double-step near-edge structure is a fingerprint of hybridization changes of the outermost orbitals between the absorbing atom and the nearest neighbors. ${ }^{53,54}$ In this way, we observe that the intensity of this spectral feature enhances as the sintering temperature does. This result indicates that the hybridization of $\mathrm{Cu}$ with the neighboring atoms is reinforced by the sintering. Further confirmation of this result can be obtained from the behavior of the white-line (peak $B$ ) intensity. The intensity of the white line is proportional to the number of empty states of the allowed symmetry projected on the absorption site. The comparison of the $\mathrm{x}$-ray absorption in the gaseous state with the absorption in the condensed metallic state shows that this intensity is a measure of the localization of these states..$^{53,54,59}$ The comparison of the XANES spectra of the samples studied in this work shows that there is a progressive reduction of the white-line intensity as the temperature of sintering increases, being the highest for the as-milled samples $(B M)$. These results indicate that, despite the fact that the local structure is preserved in all the cases, the sintering influences the hybridization of the conduction states of $\mathrm{Cu}$ atoms with their neighbors. In this way, $\mathrm{Cu}$ conduction states are more localized in the as-milled samples and then their hybridization with $\mathrm{O}$ is reduced. As the samples are sintered, this hybridization increases and, consequently, the capability of $\mathrm{O}$ to trap the conducting holes is reduced. The picture emerging from XAS analysis confirms that the conductivity of the samples increases after the sintering because of the modification of the localization (hybridization) of the conduction states, while the crystal structure remains unchanged.

We have studied the impurity phase content and the occurrence of chemical disorder in our samples from the XANES spectra. To this end, we have performed the ab initio computation of the $\mathrm{Cu} K$-edge XANES for $\mathrm{CuAl}_{2} \mathrm{O}_{4}$ and for different $\mathrm{CuAlO}_{2}$ clusters in which the occupation of the crystallographic positions of both $\mathrm{Cu}$ and $\mathrm{Al}$ atoms have been exchanged. Our first objective has been to determine the necessary conditions to obtain an accurate reproduction of the experimental spectra in these compounds to fix the same computational parameters for the computation of the other systems. To achieve this, we have first computed the $\mathrm{Cu} K$-edge spectrum in the case of the reference compound $\mathrm{Cu}_{2} \mathrm{O}$. For these computations, we have built up a $\mathrm{Cu}_{2} \mathrm{O}$ cluster containing 127 atoms, i.e., including contributions from atoms located within the first $\sim 7.4 \AA$ around $\mathrm{Cu}^{6}{ }^{60}$ We have determined by using this cluster that the best agreement, both in the relative energy and intensity of the different spectral features, between the experimental and theoretical $\mathrm{Cu} K$-edge absorption spectra is obtained by using the energy-dependent Dirac-Hara (DH) exchange and correlation potential (ECP) (see Fig. 8). The fact that the DH potential gives overall better agreement with the experiments than the most frequently used Hedin-Lundqvist (HL) potential has been previously found at the $K$ edge of different $3 d$ systems. ${ }^{27-29,61}$ More interestingly, these results indicate that single-channel multiple-scattering calculations are capable of reproducing the experimental $\mathrm{Cu}$ $K$-edge spectra of $\mathrm{Cu}(\mathrm{I})$ systems. By contrast, two absorption channels, associated with the $3 d^{9}$ and $3 d^{10} \underline{L}$ ( $\underline{L}$ being a hole from ligand) electronic configurations, are needed to account for a proper description of the final state during the photoabsorption process in $\mathrm{Cu}$ (II) systems $\mathrm{s}^{30,32,33}$ as the $\mathrm{CuO}$ reference. $^{31}$

Following these prescriptions, we have calculated the $\mathrm{Cu}$ $K$-edge XANES spectrum of $\mathrm{CuAlO}_{2}$. In this way, computations have been made for a cluster containing 129 atoms, which includes the scattering contributions from neighboring atoms within the first $7.2 \AA$ around photoabsorbing $\mathrm{Cu}$. As shown in Fig. 9, a good agreement is found between 


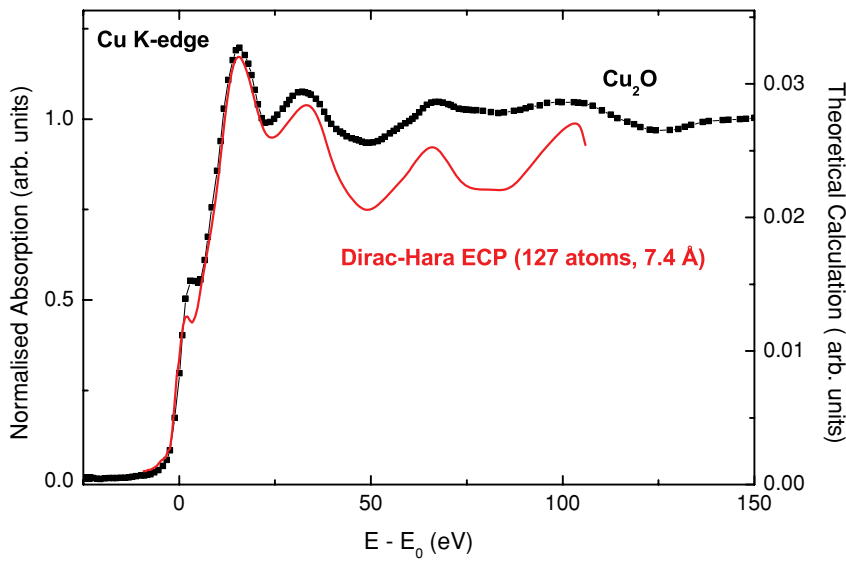

FIG. 8. (Color online) Comparison of the $\mathrm{Cu} K$-edge XANES spectrum of $\mathrm{Cu}_{2} \mathrm{O}$ (bullet) and the theoretical spectrum calculated on a 127 -atom $(7.4 \AA$ ) cluster by using the Dirac-Hara ECP potential (red, solid line).

the experimental and theoretical spectra. The next step in our study is to determine if the content of the residual $\mathrm{CuAl}_{2} \mathrm{O}_{4}$ phase is modified by the sintering process, thus giving rise to the different electrical conductivity behavior observed in the studied samples. With this in mind, we have constructed a $\mathrm{CuAl}_{2} \mathrm{O}_{4}$ cluster covering the first $7.4 \AA$ around photoabsorbing $\mathrm{Cu}$. It should be noted that, because $\mathrm{Cu}$ occupies two different crystallographic positions, i.e., $8 a$ and $16 d$, XANES computations have to be performed for two different clusters in which the absorbing $\mathrm{Cu}$ atom lies at the $8 a(\mathrm{Cu} 1)$ and at the $16 d(\mathrm{Cu} 2)$ positions, respectively. ${ }^{62}$ Then, the theoretical spectra are made up from the weighted sum of both contributions according the crystallographic 1:2 statistical ratio. For these computations, the $\mathrm{CuAl}_{2} \mathrm{O}_{4}$ clusters centered at $\mathrm{Cu} 1$ and $\mathrm{Cu} 2$ contain, respectively, 173 and 179 atoms. The result of these calculations are compared to those of $\mathrm{CuAlO}_{2}$ in Fig. 9. The computation for $\mathrm{CuAl}_{2} \mathrm{O}_{4}$ yields a positive contribution at the same energy, $\sim 27 \mathrm{eV}$, at which both the theoretical and experimental $\mathrm{CuAlO}_{2}$ spectra exhibit

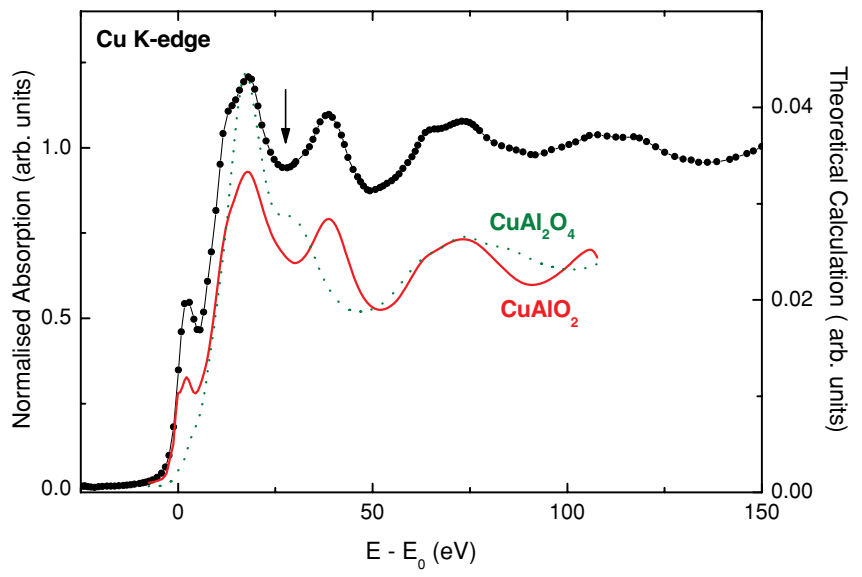

FIG. 9. (Color online) Comparison of the $\mathrm{Cu} K$-edge XANES spectrum of the $M I C R O \mathrm{CuAlO}_{2}$ sample (bullets) and the theoretical spectra calculated on both a $7.2-\AA \mathrm{CuAlO}_{2}$ (red, solid line) and a 7.4- $\AA \mathrm{CuAl}_{2} \mathrm{O}_{4}$ (green, dots) clusters by using the Dirac-Hara ECP potential (red, solid line).
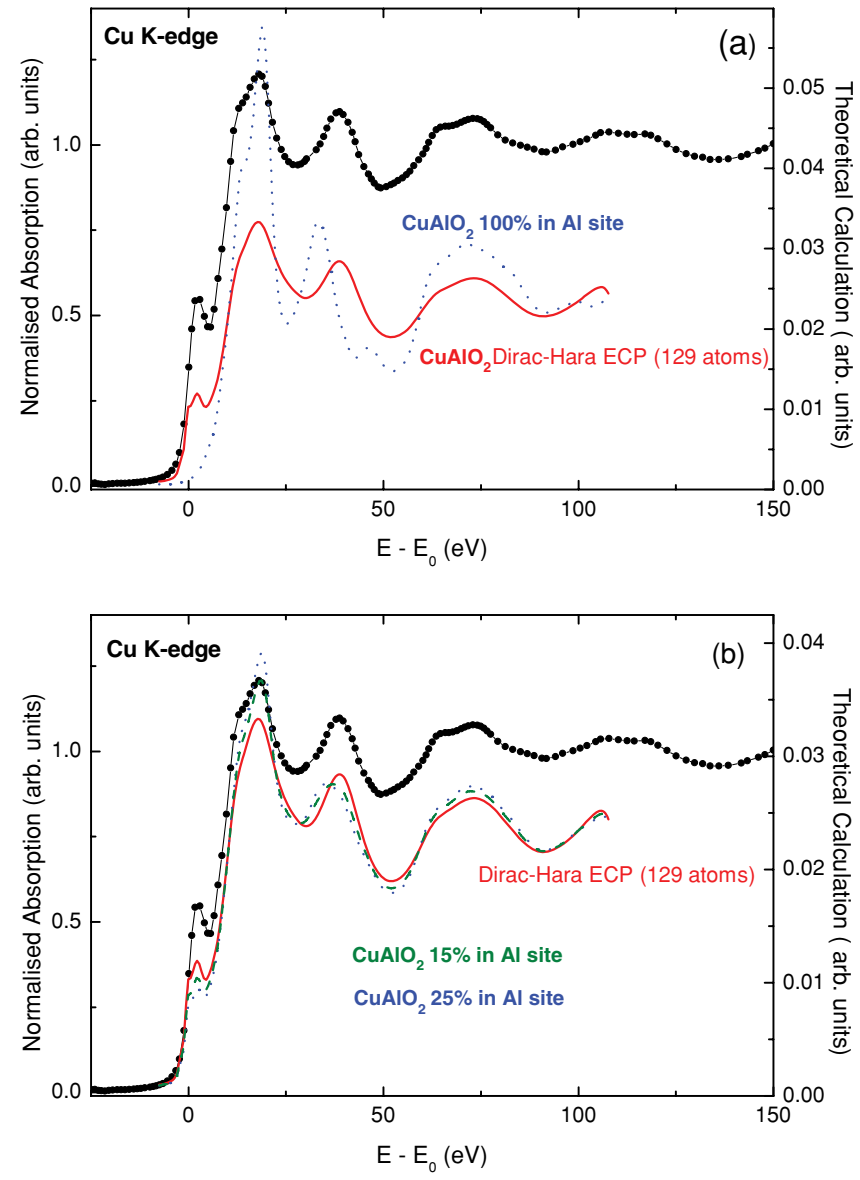

FIG. 10. (Color online) (a) Comparison of the $\mathrm{Cu} K$-edge XANES experimental spectra of the $M I C R O \mathrm{CuAlO}_{2}$ sample (filled bullet) and the theoretical spectra calculated for an ordered $\mathrm{CuAlO}_{2}$ cluster (red, solid line) and for a second one (blue, dot line) in which all the neighboring $\mathrm{Cu}$ and $\mathrm{Al}$ atoms around the photoabsorbing $\mathrm{Cu}$ have exchanged their crystallographic positions (see text for details). (b) Same comparison as above for $\mathrm{CuAlO}_{2}$ clusters in which the chemical disordering extends to $25 \%$ (green, dash line) and $15 \%$ (blue, dot line) of the $\mathrm{Cu}$ atoms.

the first energy minimum. In addition, the calculated spectra of $\mathrm{CuAl}_{2} \mathrm{O}_{4}$ shows a broad negative deep which extends over the energy region in which $\mathrm{CuAlO}_{2}$ shows the first positive peak, $\sim 38 \mathrm{eV}$, and the second energy minimum, $\sim 50 \mathrm{eV}$. These results allow us to exclude the modification of the $\mathrm{CuAl}_{2} \mathrm{O}_{4}$ content in the samples upon sintering. Indeed, although a certain amount of this phase was present in alloys, if its concentration varies during sintering, we would expect to observe clear changes of the spectral shape in the energy region extending between 25 and $50 \mathrm{eV}$ above the edge. By contrast, the experimental results do not show this behavior, which led us to conclude that the modification of the electrical conductivity in the $N A N O$ samples is an intrinsic effect that is not linked to the presence of impurity phases.

Finally, we have also considered the possibility that the mechanical milling process introduced chemical disordering in the samples. In this way, some of the $\mathrm{Cu}$ and $\mathrm{Al}$ atoms may exchange their crystallographic position within the $\mathrm{CuAlO}_{2}$ matrix. High-temperature sintering could induce the reordering of these exchanged atoms, leading to the modification 
of the electrical conductivity. To assess this possibility, we have constructed several $\mathrm{CuAlO}_{2}$ clusters in which some $\mathrm{Cu}$ and $\mathrm{Al}$ have exchanged their position. The results of these computations are reported in Fig. 10. The main result is that the $\mathrm{Cu}-\mathrm{Al}$ exchange mainly affects the intensity of the white line and the spectral shape in the region covering the first negative dip (centered at $\sim 25 \mathrm{eV}$ ) and the first positive peak $(\sim 38 \mathrm{eV})$. We have evaluated this effect by reducing progressively the percent of $\mathrm{Cu}$ and $\mathrm{Al}$ atoms that interchange their crystallographic position. As shown in the lower panel of Fig. 10, even for a low exchange ratio, the expected peak positions should be shifted by about $4 \mathrm{eV}$ with respect to those of ordered $\mathrm{CuAlO}_{2}$. Such variations involving the energy shift of the energy minima and maxima should lead to a modification of the spectral shape, which could be clearly distinguished in the comparison of the experimental spectra recorded on the samples with different sintering treatment. However, the comparison of the theoretical and experimental results reported, respectively, in Figs. 10 and 7 does not support this hypothesis.

\section{SUMMARY AND CONCLUSIONS}

We have reported in this work the electrical, thermoelectrical, thermal, and structural characterization of nanocrystalline and microcrystalline samples of $\mathrm{CuAlO}_{2}$ with delaffosite structure. We have found significant differences between the electrical and thermal properties. Our results display a large discrepancy between the activation energies associated to thermoelectric power $E_{S}$ and a significant reduction of the electrical conductivity for the nanocrystalline sample. In view of the results presented here, the changes in $\sigma(T)$ and $S(T)$ can be attributed to the narrowing of the polaron band gap as the grain size decreases. The disorder introduced in the nanocrystal line sample enhances the localization of charge carriers associated to $\mathrm{Cu}$ atoms. As a consequence, the electrical conductivity caused by hopping of small polarons falls several orders of magnitude down in the case of nanocrystalline sample.

On the other hand, we find a reduction of the thermal conductivity in the nanocrystalline sample due to grain boundaries that behave like barriers to the phonon transport and induce phonon localization. However, in spite of thermal-conductivity reduction, since electrical conductivity is largely reduced by decreasing the grain size, mechanical alloying does not improve, at least in $\mathrm{CuAlO}_{2}$, its properties for thermoelectric applications.

Finally, the combined experimental and theoretical study of the $\mathrm{Cu} K$-edge XAS spectra of the mechanically milled $\mathrm{CuAlO}_{2}$ samples presented indicates that the local structure around $\mathrm{Cu}$ is preserved upon the sintering process. Moreover, these results indicate that the observed behavior of the electrical conductivity is of intrinsic origin, not associated to the presence of impurity phases or to the $\mathrm{Cu}-\mathrm{Al}$ chemical disorder. The analysis of the near-edge region of the absorption spectra indicates that sintering favors the $\mathrm{Cu}-\mathrm{O}$ hybridization. As a result, oxygen atoms progressively lose their capability of trapping holes, thereby improving the electrical conductivity, as shown by our experimental results.

\section{ACKNOWLEDGMENTS}

We acknowledge financial support by JCCM through Projects No. PAI-05-013 and PCI08-0091, and the Spanish CICYT through Grants No. MAT2008-06542-C04-01 and MAT2008-06517-C02. R.B. acknowledges support from the Ministerio de Ciencia e Innovación of Spain. We are grateful to J. Santamaría for helpful discussions, and to L. Vázquez for the AFM images. The assistance of the SpLine staff during the experimental work at ESRF is acknowledged.
${ }^{1}$ B. C. Sales, D. Mandrus, and R. K. Williams, Science 272, 1325 (1996).

${ }^{2}$ G. S. Nolas, J. L. Cohn, G. A. Slack, and S. B. Schujman, Appl. Phys. Lett. 73, 178 (1998).

${ }^{3}$ K. Mastronardi, D. Young, C. C. Wang, P. Khalifah, and R. J. Cava, Appl. Phys. Lett. 74, 1415 (1999).

${ }^{4}$ R. Robert, S. Romer, A. Reller, and A. Weidenkaff, Adv. Eng. Mater 7, 303 (2005).

${ }^{5}$ B. Poudel et al., Science 324, 620 (2008).

${ }^{6}$ G. Chen, M. S. Dresselhaus, G. Dresselhaus, J. P. Fleurial, and T. Caillat, Int. Mater. Rev. 48, 45 (2003).

${ }^{7}$ H. Kawazoe, M. Yasukawa, H. Hyodo, M. Kurita, H. M. Yanagi, and H. Hosono, Nature (London) 389, 939 (1997).

${ }^{8}$ B. J. Ingram, T. O. Mason, R. Asahi, K. T. Park, and A. J. Freeman, Phys. Rev. B 64, 155114 (2001).

${ }^{9}$ A. N. Banerjee and K. K. Chattopadhyay, Prog. Cryst. Growth Charact. Mater. 53, 52 (2005).

${ }^{10}$ K. Koumoto, H. Koduka, and W.-S. Seo, J. Mater. Chem. 11, 251 (2001).

${ }^{11}$ I. G. Austin and N. F. Mott, Adv. Phys. 50, 757 (2001).

${ }^{12}$ T. Holstein, Ann. Phys. (NY) 8, 343 (1959).
${ }^{13}$ D. Emin, Phys. Today 35, 34 (1982).

${ }^{14}$ P. A. Lee and J. B. Pendry, Phys. Rev. B 11, 2795 (1975).

${ }^{15}$ H. Kawazoe, M. Yasukawa, H. Hyodo, M. Kurita, H. Yanagi, and H. Hosono, Nature (London) 389, 939 (1997).

${ }^{16} \mathrm{H}$. Yanagi, S. Inoue, K. Ueda, H. Kawazoe, H. Hosono, and N. Hamada, J. Appl. Phys. 88, 4159 (2000).

${ }^{17}$ J. Tate, H. L. Ju, J. C. Moon, A. Zakutayev, A. P. Richard, J. Russell, and D. H. McIntyre, Phys. Rev. B 80, 165206 (2009).

${ }^{18}$ L. I. Clarke and R. Taylor, J. Appl. Phys. 46, 714 (1975).

${ }^{19}$ P. G. Klemens, High Temp. High Press. 23, 241 (1991).

${ }^{20}$ D. E. Sayers and B. A. Bunker, X-Ray Absorption: Principles, Applications, Techniques of EXAFS, SEXAFS, and XANES (Wiley, New York, 1988), Chap. 6.

${ }^{21}$ B. Ravel and M. Newville, J. Synchrotron Radiat. 12, 537 (2005).

${ }^{22}$ M. S. del Río, J. Chaboy, and R. Djerbi, Comput. Chem. (Oxford, UK) 19, 59 (1995).

${ }^{23}$ C. R. Natoli and M. Benfatto (unpublished).

${ }^{24}$ C. R. Natoli and M. Benfatto, J. Phys., Colloq. 47, C8-11 (1986).

${ }^{25}$ J. Chaboy and S. Quartieri, Phys. Rev. B 52, 6349 (1995).

${ }^{26}$ J. Chaboy, J. Synchrotron Radiat. 16, 533 (2009). 
${ }^{27}$ J. Chaboy, H. Maruyama, and N. Kawamura, J. Phys. Condens. Matter 19, 216214 (2007).

${ }^{28}$ J. Chaboy, N. Nakajima, and Y. Tezuka, J. Phys. Condens. Matter 19, 266206 (2007).

${ }^{29}$ K. Hatada and J. Chaboy, Phys. Rev. B 76, 104411 (2007).

${ }^{30}$ J. Chaboy, A. Muñoz-Páez, F. Carrera, P. Merkling, and E. Sánchez Marcos, Phys. Rev. B 71, 134208 (2005).

${ }^{31}$ J. Chaboy, A. Muñoz-Páez, and E. Sánchez-Marcos, J. Synchrotron Radiat. 13, 471 (2006).

${ }^{32}$ F. Carrera, E. Sánchez-Marcos, P. J. Merkling, J. J. Chaboy, and A. Muñoz-Páez, Inorg. Chem. 43, 6674 (2004).

${ }^{33}$ J. Chaboy, A. Muñoz-Páez, P. J. Merkling, and E. Sánchez-Marcos, J. Chem. Phys. 124, 064509 (2006).

${ }^{34}$ M. O. Krause and J. H. Oliver, J. Phys. Chem. Ref. Data 8, 329 (1979).

${ }^{35}$ N. F. Mott and E. A. Davis, Electronic Process in Non Crystalline Materials (Clarendon Press, Oxford, 1971).

${ }^{36}$ A. K. Jonscher, Dielectric Relaxation in Solids (Chelsea Dielectrics, London, 1983).

${ }^{37}$ J. C. Dyre and T. B. Schrøder, Rev. Mod. Phys. 72, 873 (2000).

${ }^{38}$ C. León, M. L. Lucía, J. Santamaría, and F. Sánchez-Quesada, Phys. Rev. B 57, 41 (1998).

${ }^{39}$ K. Park, K. Y. Ko, and W.-S. Seo, J. Eur. Ceram. Soc. 25, 2219 (2005).

${ }^{40}$ M. Jaime, M. B. Salamon, M. Rubinstein, R. E. Treece, J. S. Horwitz, and D. B. Chrisey, Phys. Rev. B 54, 11914 (1996).

${ }^{41}$ R. Ang, W. J. Lu, R. L. Zhang, B. C. Zhao, X. B. Zhu, W. H. Song, and Y. P. Sun, Phys. Rev. B 72, 184417 (2005).

${ }^{42}$ S. Wang, K. Li, Z. Chen, and Y. Zhang, Phys. Rev. B 61, 575 (2000).

${ }^{43}$ T. Okuda, Y. Beppu, Y. Fujii, T. Onoe, N. Terada, and S. Miyasaka, Phys. Rev. B 77, 134423 (2008).

${ }^{44}$ D. G. Cahill, S. K. Watson, and R. O. Pohl, Phys. Rev. B 46, 6131 (1992).

${ }^{45}$ O. J. Durá, E. Bauer, L. Vázquez, and M. A. López de la Torre, J. Phys. D 43, 105407 (2010).
${ }^{46}$ Y. Wang, K. Fujinami, R. Zhang, C. Wan, N. Wang, Y. Ba, and K. Koumoto, Appl. Phys. Express 3, 031101 (2010).

${ }^{47}$ L. Braginsky, N. Lukzen, V. Shklover, and H. Hofmann, Phys. Rev. B 66, 134203 (2002).

${ }^{48}$ A. L Ankudinov and J. J. Rehr, Phys. Rev. B 62, 2437 (2000).

${ }^{49}$ P. H. Citrin, P. Eisenberger, and B. M. Kincaid, Phys. Rev. Lett. 36, 1346 (1976).

${ }^{50}$ L. Downward, F. Bridges, S. Bushart, J. J. Neumeier, N. Dilley, and L. Zhou, Phys. Rev. Lett. 95, 106401 (2005).

${ }^{51}$ F. Bridges, L. Downward, J. J. Neumeier, and T. A. Tyson, Phys. Rev. B 81, 184401 (2010).

${ }^{52}$ S. I. Ahmed, G. Dalba, P. Fornasini, M. Vaccari, F. Rocca, A. Sanson, J. Li, and A. W. Sleight, Phys. Rev. B 79, 104302 (2009).

${ }^{53}$ J. Chaboy, A. Marcelli, and L. Bozukov, J. Phys. Condens. Matter 7, 8197 (1995).

${ }^{54}$ J. Chaboy, J. García, and A. Marcelli, J. Magn. Magn. Mater. 166, 149 (1997).

${ }^{55}$ J. Chaboy, H. Maruyama, L. M. García, J. Bartolomé, K. Kobayashi, N. Kawamura, A. Marcelli, and L. Bozukov, Phys. Rev. B 54, R15637 (1996).

${ }^{56}$ J. Chaboy, L. M. García, F. Bartolomé, H. Maruyama, A. Marcelli, and L. Bozukov, Phys. Rev. B 57, 13386 (1998).

${ }^{57}$ J. Chaboy, A. Marcelli, L. Bozukov, F. Baudelet, E. Dartyge, A. Fontaine, and S. Pizzini, Phys. Rev. B 51, 9005 (1995).

${ }^{58}$ J. Chaboy, M. A. Laguna-Marco, M. C. Sánchez, H. Maruyama, N. Kawamura, and M. Suzuki, Phys. Rev. B 69, 134421 (2004).

${ }^{59}$ J. I. Espeso, J. C. Gómez Sal, and J. Chaboy, Phys. Rev. B 63, 014416 (2000).

${ }^{60}$ R. Restori and D. Schwarzenbach, Acta Crystallogr. Sect. A 42, 201 (1986).

${ }^{61}$ K. Hatada, K. Hayakawa, J. Chaboy, and C. R. Natoli, J. Phys.: Conf. Ser. 190, 012010 (2009).

${ }^{62}$ H. S. C. O'Neill, M. James, W. A. Dollase, and S. A. T. Redfern, European J. Miner. 17, 581 (2005). 